\title{
Optimizing the removal of humic acid with polyaluminum chloride and polyaluminum ferric chloride as green coagulants using response surface methodology
}

\author{
Maryam Faraji ${ }^{\mathrm{a}}$, Afshin Ebrahimi ${ }^{\mathrm{b}}$, Heshmatollah Nourmoradi ${ }^{\mathrm{c}, \mathrm{d}}$, Ali Nikoonahad ${ }^{\mathrm{d}}$, \\ Ali Abdolahnejade, Reza Ghanbari ${ }^{\mathrm{f}}$, Amir Mohammadig,** \\ ${ }^{a}$ Environmental Health Engineering Research Center, Kerman University of Medical Sciences, Kerman, Iran \\ ${ }^{b}$ Environment Research Center, Research Institute for Primordial Prevention of Non-Communicable Disease, Isfahan University \\ of Medical Sciences, Isfahan, Iran, email: a_ebrahimi@hlth.mui.ac.ir \\ 'Biotechnology and Medical Plants Research Center, Ilam University of Medical Sciences, Ilam, Iran \\ ${ }^{d}$ Department of Environmental Health Engineering, School of Health, Ilam University of Medical Sciences, Ilam, Iran \\ ${ }^{e}$ Department of Environmental Health Engineering, Environmental Science and Technology Research Center, Shahid Sadoughi \\ University of Medical Sciences, Yazd, Iran \\ fSocial Determinants of Health Research Center, Qazvin University of Medical Sciences, Qazvin, Iran \\ sDepartment of Public Health, School of Nursing and Midwifery, Maragheh University of Medical sciences, Maragheh, Iran, \\ Tel.+98-9141860599, email: mohammadiurm@gmail.com(A. Mohammadi)
}

Received 26 May 208; Accepted 16 October 2018

\begin{abstract}
A B S T R A C T
Humic acid is a major precursor of chlorinated byproducts that need to be removed from water, via treatment, given their adverse effects on human health. The current study aimed at identifying the optimal conditions to remove humic acid from surface water, using coagulants such as polyaluminum chloride (PACl) and polyaluminum ferric chloride ( $\mathrm{PAFCl})$. The effects of independent variables such as total organic carbon (TOC) concentration (1.6-7 mg/L), pH level (5-9), and coagulant dosage $(10-50 \mathrm{mg} / \mathrm{L})$ on humic acid removal were studied using response surface methodology and central composite design. A coagulant dose of $15.72 \mathrm{mg} / \mathrm{L}$ (for PACl and PAFCl), TOC concentration of $2.66 \mathrm{mg} / \mathrm{L}$, and $\mathrm{pH}$ of 7.84 comprised the optimal conditions, and the removal efficiencies of $97.55 \%$ and $98.18 \%$ were obtained with $\mathrm{PACl}$ and $\mathrm{PAFCl}$, respectively. Zeta potential analysis showed that the leading mechanism for coagulation, with both coagulants, was charge neutralization. PACl and $\mathrm{PAFCl}$ had adequate potential to remove humic acid from surface water, but PAFCl had a better performance regarding the treatment of aluminum residuals.
\end{abstract}

Keywords: Coagulation; Humic acid; Polyaluminum chloride; Polyaluminum ferric chloride

\footnotetext{
*Corresponding author.
} 